\title{
THE NUMBER OF TWO CONSECUTIVE SUCCESSES IN A HOPPE-PÓLYA URN
}

\author{
LARS HOLST, ${ }^{*}$ Royal Institute of Technology
}

\begin{abstract}
In a sequence of independent Bernoulli trials the probability of success in the $k$ th trial is $p_{k}=a /(a+b+k-1)$. An explicit formula for the binomial moments of the number of two consecutive successes in the first $n$ trials is obtained and some consequences of it are derived.

Keywords: Bernoulli trial; binomial moment; Hoppe urn; overlapping indicators; Pólya urn; records; random permutation
\end{abstract}

2000 Mathematics Subject Classification: Primary 60C05

Secondary 60K99

\section{Introduction}

An urn initially contains one white ball and one black ball of weight $a>0$ and $b \geq 0$, respectively. Balls are randomly drawn from the urn with probabilities proportional to weights. Every time a white ball or a black ball is drawn from the urn, it is replaced with a ball of weight 1 of a colour not already in the urn, otherwise a ball is replaced together with a copy of it. We call this drawing scheme a Hoppe-Pólya urn. If $b=0$, there is no black ball, the so-called Hoppe urn. If all balls emanating from a draw of the white or black are coloured white or, respectively, black, we obtain the well-known Pólya urn.

Let the sequence of independent Bernoulli random variables $I_{1}, I_{2}, I_{3}, \ldots$ indicate the drawings of the white ball, the 'successes' or 'records' in the Hoppe-Pólya urn. Obviously,

$$
p_{k}=\mathrm{P}\left(I_{k}=1\right)=1-\mathrm{P}\left(I_{k}=0\right)=\frac{a}{a+b+k-1}, \quad k=1,2, \ldots
$$

The number of successes in the first $n$ trials can be written as

$$
K_{n}=I_{1}+I_{2}+\cdots+I_{n},
$$

and the number of two consecutive successes can be written as

$$
M_{n}=I_{1} I_{2}+I_{2} I_{3}+\cdots+I_{n-1} I_{n} .
$$

An explicit formula for the binomial moments of $M_{n}$ is the main result of this paper. Note that $0 \leq M_{n} \leq n-1$.

For $p_{k}=a /(a+b+k-1)$, the Borel-Cantelli lemma implies that

$$
M_{\infty}=\sum_{k=1}^{\infty} I_{k} I_{k+1}<+\infty
$$

\footnotetext{
Received 21 December 2007; revision received 8 May 2008.

* Postal address: Department of Mathematics, Royal Institute of Technology, SE-100 44 Stockholm, Sweden.

Email address: 1holst@math.kth.se
} 
with probability 1 . For the case in which $a=1$ and $b=0$, i.e. $p_{k}=1 / k$, connected with record values and random permutations, Hahlin (1995) proved that $M_{\infty}$ is Poisson distributed with mean 1. After that, an unpublished proof of the same result by Diaconis inspired a number of studies on the distribution of $M_{\infty}$; see Chern et al. (2000), Mori (2001), Joffe et al. (2004), Sethuraman and Sethuraman (2004), Holst (2007), and the references therein. To the author's knowledge, the result in this paper on the distribution of $M_{n}$ for finite $n$ has not been obtained previously.

\section{Notation and facts}

Following Knuth (1992), we denote falling and rising factorials by

$$
\begin{gathered}
x^{\underline{n}}=x(x-1) \cdots(x-n+1), \\
x^{\bar{n}}=x(x+1) \cdots(x+n-1)=\sum_{j=1}^{n}\left[\begin{array}{l}
n \\
j
\end{array}\right] x^{j},
\end{gathered}
$$

where $\left[\begin{array}{c}n \\ j\end{array}\right]$ is a cycle number or signless Stirling number of the first kind. Recall the combinatorial interpretation: $\left[\begin{array}{l}n \\ j\end{array}\right]$ is the number of permutations of $1,2, \ldots, n$ with $j$ cycles.

For $K_{n}$ equals the number of successes in the first $n$ trials, we have

$$
\begin{aligned}
\mathrm{E}\left(x^{K_{n}}\right) & =\prod_{k=1}^{n}\left(\frac{a}{a+b+k-1} x+1-\frac{a}{a+b+k-1}\right) \\
& =\frac{(a x+b)^{\bar{n}}}{(a+b)^{\bar{n}}} \\
& =\sum_{j=1}^{n}\left[\begin{array}{c}
n \\
j
\end{array}\right] \frac{(a x+b)^{j}}{(a+b)^{\bar{n}}} \\
& =\sum_{i=0}^{n} x^{i} \sum_{j=i}^{n}\left[\begin{array}{c}
n \\
j
\end{array}\right]\left(\begin{array}{l}
j \\
i
\end{array}\right) \frac{a^{i} b^{j-i}}{(a+b)^{\bar{n}}} .
\end{aligned}
$$

Hence, for $i=0,1,2, \ldots n$,

$$
\mathrm{P}\left(K_{n}=i\right)=\sum_{j=i}^{n}\left[\begin{array}{l}
n \\
j
\end{array}\right] \frac{(a+b)^{j}}{(a+b)^{\bar{n}}}\left(\begin{array}{l}
j \\
i
\end{array}\right)\left(\frac{a}{a+b}\right)^{i}\left(\frac{b}{a+b}\right)^{j-i} .
$$

In particular, for $b=0$, i.e. Hoppe's urn, we obtain the cycle distribution,

$$
\mathrm{P}\left(K_{n}=i\right)=\left[\begin{array}{c}
n \\
i
\end{array}\right] \frac{a^{i}}{a^{\bar{n}}}, \quad i=1,2, \ldots, n,
$$

for an $a$-biased random permutation; see Arratia et al. (2003, p. 100).

The number of times the white ball, or balls emanating from it, is drawn in the first $n$ trials, $X_{n}$, has the following Pólya-Eggenberger distribution:

$$
\mathrm{P}\left(X_{n}=i\right)=\left(\begin{array}{l}
n \\
i
\end{array}\right) \frac{a^{\bar{i}} b^{\overline{n-i}}}{(a+b)^{\bar{n}}}=\mathrm{E}\left(\left(\begin{array}{l}
n \\
i
\end{array}\right) U^{i}(1-U)^{n-i}\right), \quad i=0,1,2, \ldots, n,
$$


where $U$ is a $\operatorname{Beta}(a, b)$ random variable with density

$$
f_{U}(u)=\frac{\Gamma(a+b)}{\Gamma(a) \Gamma(b)} u^{a-1}(1-u)^{b-1}, \quad 0<u<1 .
$$

Using the binomial distribution, we obtain, for $r=1,2, \ldots, n$,

$$
\mathrm{E}\left(\begin{array}{c}
X_{n} \\
r
\end{array}\right)=\mathrm{E}\left(\sum_{i=r}^{n}\left(\begin{array}{l}
i \\
r
\end{array}\right)\left(\begin{array}{l}
n \\
i
\end{array}\right) U^{i}(1-U)^{n-i}\right)=\mathrm{E}\left(\left(\begin{array}{l}
n \\
r
\end{array}\right) U^{r}\right)=\left(\begin{array}{l}
n \\
r
\end{array}\right) \frac{a^{\bar{r}}}{(a+b)^{\bar{r}}}
$$

Recall that a random variable $S$ with the hypergeometric distribution

$$
\mathrm{P}(S=i)=\left(\begin{array}{c}
c \\
i
\end{array}\right)\left(\begin{array}{c}
d \\
n-i
\end{array}\right) /\left(\begin{array}{c}
c+d \\
n
\end{array}\right)
$$

has the binomial moment

$$
\mathrm{E}\left(\begin{array}{l}
S \\
r
\end{array}\right)=\left(\begin{array}{l}
n \\
r
\end{array}\right) \frac{c^{-}}{(c+d)^{\underline{r}}}
$$

For an integer-valued random variable $Z \geq 0$ having a probability generating function with a radius of convergence larger than 1 , we have

$$
\mathrm{E}\left(x^{Z}\right)=\mathrm{E}\left((1+(x-1))^{Z}\right)=\sum_{r=0}^{\infty} \mathrm{E}\left(\begin{array}{l}
Z \\
r
\end{array}\right)(x-1)^{r}=\sum_{i=0}^{\infty} x^{i} \sum_{r=i}^{\infty}(-1)^{r-i}\left(\begin{array}{l}
r \\
i
\end{array}\right) \mathrm{E}\left(\begin{array}{l}
Z \\
r
\end{array}\right),
$$

which gives the following probability function of $Z$ expressed in binomial moments:

$$
\mathrm{P}(Z=i)=\sum_{r=i}^{\infty}(-1)^{r-i}\left(\begin{array}{l}
r \\
i
\end{array}\right) \mathrm{E}\left(\begin{array}{l}
Z \\
r
\end{array}\right), \quad i=0,1,2, \ldots
$$

Note that if $0 \leq Z<n$ then $\mathrm{E}\left(\begin{array}{l}Z \\ r\end{array}\right)=0$ for $r \geq n$.

\section{The number of two consecutive successes}

The following result implicitly gives the distribution of $M_{n}$.

Theorem 3.1. For $p_{k}=a /(a+b+k-1)$ and $r=1,2, \ldots, n-1$,

$$
\mathrm{E}\left(\begin{array}{c}
M_{n} \\
r
\end{array}\right)=\frac{a^{r}}{(a+b+n-1)^{r}} \sum_{k=1}^{r}\left(\begin{array}{c}
r-1 \\
r-k
\end{array}\right)\left(\begin{array}{c}
n-r \\
k
\end{array}\right) \frac{a^{\bar{k}}}{(a+b)^{\bar{k}}}
$$

Before proving the theorem we consider the special case $b=0$, i.e. Hoppe's urn. A more general result is Proposition 3 of Holst (2007).

Lemma 3.1. For $p_{k}=a /(a+k-1)$ and $r=1,2, \ldots, n-1$,

$$
\mathrm{E}\left(\begin{array}{c}
M_{n} \\
r
\end{array}\right)=\left(\begin{array}{c}
n-1 \\
r
\end{array}\right) \frac{a^{r}}{(a+n-1)^{\underline{r}}} .
$$


Proof. For $N_{n}=M_{n}+I_{n}$, we have

$$
\mathrm{E}\left(t^{N_{n+1}}\right)=p_{n+1} \mathrm{E}\left(t^{N_{n}}\right) t+\left(1-p_{n+1}\right) \mathrm{E}\left(t^{M_{n}}\right),
$$

which implies that

$$
\mathrm{E}\left(\begin{array}{c}
N_{n+1} \\
r
\end{array}\right)=p_{n+1}\left(\mathrm{E}\left(\begin{array}{c}
N_{n} \\
r
\end{array}\right)+\mathrm{E}\left(\begin{array}{c}
N_{n} \\
r-1
\end{array}\right)\right)+\left(1-p_{n+1}\right) \mathrm{E}\left(\begin{array}{c}
M_{n} \\
r
\end{array}\right) .
$$

For $p_{k}=a /(a+k-1)$, the random variable $N_{n}$ has the same distribution as the number of fix-points in an $a$-biased random permutation of $1,2, \ldots, n$, and

$$
\mathrm{E}\left(\begin{array}{c}
N_{n} \\
r
\end{array}\right)=\left(\begin{array}{l}
n \\
r
\end{array}\right) \frac{a^{r}}{(a+n-1) \underline{r}}
$$

see Arratia et al. (2003 pp. 95-96). Using this and the relation above, proves the assertion.

Proof of Theorem 3.1. Consider the Hoppe-Pólya urn and the random variable $X_{n}$ introduced in Section 2. In the $X_{n}$ 'white' drawings, the probability of obtaining the white ball in the $j$ th trial is

$$
p_{j}^{*}=\frac{a}{a+j-1} .
$$

Given $X_{n}=x$, the number of times the white ball is consecutively drawn in these 'white' drawings, $M_{x}^{*}$, is distributed as in Lemma 3.1.

Conditional on $X_{n}=x$, we can argue as follows. Among the $x$ 'white' draws let $W_{1}$ denote a drawing that gives a white ball and let $W_{0}$ denote a drawing that gives a ball which emanates from a white ball. Let $B$ denote a 'black' drawing. The result of the 'white' draws can be written as $W_{1} W_{i_{2}} W_{i_{3}} \cdots W_{i_{x}}$, where $i_{2}, \ldots, i_{x}$ are 0 or 1 . For $M_{x}^{*}=y, y$ of the pairs $W_{1} W_{i_{2}}, \ldots, W_{i_{x}-1} W_{i_{x}}$ are of type $W_{1} W_{1}$. For $M_{n}=z$ consecutive draws $W_{1} W_{1}$ among the original $n$ draws (with $x W_{\mathrm{s}}$ and $n-x B \mathrm{~s}$ ), there are $z$ pairs of the $y W_{1} W_{1}$-pairs among the 'white' draws which are intact and $y-z$ which are split by at least one $B$ between $W_{1} W_{1}$. The number of ways to choose the pairs to be intact is $\left(\begin{array}{l}y \\ z\end{array}\right)$. After such a splitting, there are $x-z$ 'free' $W$ s to combine with the $n-x-(y-z)$ 'free' $B$ s, and there are $\left(\begin{array}{l}n-y \\ x-z\end{array}\right)$ such combinations. As each combination of $x W \mathrm{~s}$ and $n-x B$ s has the same probability, $1 /\left(\begin{array}{l}n \\ x\end{array}\right)$, we obtain

$$
\mathrm{P}\left(M_{n}=z \mid X_{n}=x\right)=\sum_{y} \mathrm{P}\left(M_{x}^{*}=y\right)\left(\begin{array}{l}
y \\
z
\end{array}\right)\left(\begin{array}{l}
n-y \\
x-z
\end{array}\right) /\left(\begin{array}{l}
n \\
x
\end{array}\right) .
$$

Thus, $M_{n}$ 's probability function can be written as

$$
\mathrm{P}\left(M_{n}=z\right)=\sum_{x, y} \mathrm{P}\left(X_{n}=x\right) \mathrm{P}\left(M_{x}^{*}=y\right)\left(\begin{array}{l}
y \\
z
\end{array}\right)\left(\begin{array}{l}
n-y \\
x-z
\end{array}\right) /\left(\begin{array}{l}
n \\
x
\end{array}\right)
$$

with the binomial moment

$$
\mathrm{E}\left(\begin{array}{c}
M_{n} \\
r
\end{array}\right)=\sum_{x, y} \mathrm{P}\left(X_{n}=x\right) \mathrm{P}\left(M_{x}^{*}=y\right) \sum_{z}\left(\begin{array}{l}
z \\
r
\end{array}\right)\left(\begin{array}{l}
y \\
z
\end{array}\right)\left(\begin{array}{l}
n-y \\
x-z
\end{array}\right) /\left(\begin{array}{l}
n \\
x
\end{array}\right) .
$$


Using the formula for the binomial moment of the hypergeometric distribution and Lemma 3.1, we obtain

$$
\begin{aligned}
\mathrm{E}\left(\begin{array}{c}
M_{n} \\
r
\end{array}\right) & =\sum_{x, y} \mathrm{P}\left(X_{n}=x\right) \mathrm{P}\left(M_{x}^{*}=y\right)\left(\begin{array}{l}
x \\
r
\end{array}\right) \frac{y^{\underline{r}}}{n^{\underline{r}}} \\
& =\sum_{x}\left(\begin{array}{l}
x \\
r
\end{array}\right)\left(\begin{array}{l}
n \\
r
\end{array}\right)^{-1} \mathrm{P}\left(X_{n}=x\right) \sum_{y}\left(\begin{array}{l}
y \\
r
\end{array}\right) \mathrm{P}\left(M_{x}^{*}=y\right) \\
& =\sum_{x}\left(\begin{array}{l}
x \\
r
\end{array}\right)\left(\begin{array}{l}
n \\
r
\end{array}\right)^{-1}\left(\begin{array}{l}
n \\
x
\end{array}\right) \frac{a^{\bar{x}} b^{\overline{n-x}}}{(a+b)^{\bar{n}}}\left(\begin{array}{c}
x-1 \\
r
\end{array}\right) \frac{a^{r}}{(a+x-1)^{\underline{r}}} .
\end{aligned}
$$

Hence, the binomial moment of the Pólya-Eggenberger distribution gives

$$
\begin{aligned}
\mathrm{E}\left(\begin{array}{c}
M_{n} \\
r
\end{array}\right) & =\frac{a^{r}}{(a+b+n-1)^{\underline{r}}} \sum_{x}\left(\begin{array}{l}
n-r \\
x-r
\end{array}\right) \frac{a^{\overline{x-r}} b^{\overline{n-r-(x-r)}}}{(a+b)^{\overline{n-r}}} \sum_{k=1}^{r}\left(\begin{array}{c}
r-1 \\
r-k
\end{array}\right)\left(\begin{array}{c}
x-r \\
k
\end{array}\right) \\
& =\frac{a^{r}}{(a+b+n-1)^{\underline{r}}} \sum_{k=1}^{r}\left(\begin{array}{l}
r-1 \\
r-k
\end{array}\right) \sum_{t}\left(\begin{array}{l}
t \\
k
\end{array}\right)\left(\begin{array}{c}
n-r \\
t
\end{array}\right) \frac{a^{\bar{t}} b^{\overline{n-r-t}}}{(a+b)^{\overline{n-r}}} \\
& =\frac{a^{r}}{(a+b+n-1)^{\underline{r}}} \sum_{k=1}^{r}\left(\begin{array}{c}
r-1 \\
r-k
\end{array}\right) \mathrm{E}\left(\begin{array}{c}
X_{n-r} \\
k
\end{array}\right) \\
& =\frac{a^{r}}{(a+b+n-1)^{\underline{r}}} \sum_{k=1}^{r}\left(\begin{array}{c}
r-1 \\
r-k
\end{array}\right)\left(\begin{array}{c}
n-r \\
k
\end{array}\right) \frac{a^{\bar{k}}}{(a+b)^{\bar{k}}},
\end{aligned}
$$

which proves the assertion.

The distribution of $M_{\infty}$ was obtained by Mori (2001). It is a special case of the distribution given in Theorem 1 of Holst (2007).

Corollary 3.1. Conditional on a Beta $(a, b)$ random variable $U, M_{\infty}$ is Poisson distributed with mean $a U$.

Proof. From Theorem 3.1, it follows that

$$
\mathrm{E}\left(\begin{array}{c}
M_{n} \\
r
\end{array}\right) \rightarrow \frac{a^{r}}{r !} \frac{a^{\bar{r}}}{(a+b)^{\bar{r}}}, \quad n \rightarrow \infty .
$$

As $\mathrm{E}\left(U^{r}\right)=a^{\bar{r}} /(a+b)^{\bar{r}}$, we obtain, using the Poisson distribution,

$$
\mathrm{E}\left(\begin{array}{c}
M_{\infty} \\
r
\end{array}\right)=\mathrm{E}\left(\mathrm{E}\left(\left(\begin{array}{c}
M_{\infty} \\
r
\end{array}\right) \mid U\right)\right)=\mathrm{E}\left(\frac{(a U)^{r}}{r !}\right)=\frac{a^{r} \mathrm{E}\left(U^{r}\right)}{r !}=\frac{a^{r}}{r !} \frac{a^{\bar{r}}}{(a+b)^{\bar{r}}}
$$

The assertion follows from the moment convergence.

The distribution of $M_{n}$ for $p_{k}=p$ was studied by Hirano et al. (1991); see also the references therein. Letting $a, b \rightarrow \infty$ such that $a /(a+b) \rightarrow p$, we obtain their result.

Corollary 3.2. For $p_{k}=p$ and $r=1,2, \ldots, n-1$,

$$
\mathrm{E}\left(\begin{array}{c}
M_{n} \\
r
\end{array}\right)=p^{r} \sum_{k=1}^{r}\left(\begin{array}{c}
r-1 \\
r-k
\end{array}\right)\left(\begin{array}{c}
n-r \\
k
\end{array}\right) p^{k}
$$


Finally, consider the Pólya urn starting with one white ball of weight $a$ and one black ball of weight $b$. Every drawn ball is replaced together with one ball of the same colour and of weight 1 . In $n$ drawings, the number of times a white ball is drawn, $X_{n}$, has the PólyaEggenberger distribution. Let $Y_{n}$ denote the number of times a white ball is consecutively drawn.

Corollary 3.3. For the Pólya urn and $r=1,2, \ldots, n-1$,

$$
\mathrm{E}\left(\begin{array}{c}
Y_{n} \\
r
\end{array}\right)=\sum_{k=1}^{r}\left(\begin{array}{c}
r-1 \\
r-k
\end{array}\right)\left(\begin{array}{c}
n-r \\
k
\end{array}\right) \frac{a^{\overline{r+k}}}{(a+b)^{\overline{r+k}}} .
$$

Proof. Set $J_{k}=1$ if the $k$ th drawn ball is white, otherwise set $J_{k}=0$. It is a well known, easily proved fact that, conditional on a $\operatorname{Beta}(a, b)$ random variable $U$, the random variables $J_{1}, J_{2}, \ldots$ are independent and Bernoulli distributed with success probability $U$. Thus, it follows from Corollary 3.2 that

$$
\mathrm{E}\left(\begin{array}{c}
Y_{n} \\
r
\end{array}\right)=\mathrm{E}\left(U^{r} \sum_{k=1}^{r}\left(\begin{array}{c}
r-1 \\
r-k
\end{array}\right)\left(\begin{array}{c}
n-r \\
k
\end{array}\right) U^{k}\right)=\sum_{k=1}^{r}\left(\begin{array}{c}
r-1 \\
r-k
\end{array}\right)\left(\begin{array}{c}
n-r \\
k
\end{array}\right) \mathrm{E}\left(U^{r+k}\right),
$$

which proves the assertion.

\section{References}

Arratia, R., Barbour, A. D. and Tavaré, S. (2003). Logarithmic Combinatorial Structures: A Probabilistic Approach. European Mathematical Society, Zürich.

Chern, H.-H., Hwang, H.-K. AND Yeh, Y.-N. (2000). Distribution of the number of consecutive records. Random Structures Algorithms 17, 169-196.

Hahlin, L. O. (1995). Double records. Res. Rep. 1995:12, Department of Mathematics, Uppsala University.

Hirano, K., AKI, S., Kashiwagi, N. And KuboKi, H. (1991). On Ling's binomial and negative binomial distributions of order k. Statist. Prob. Lett. 11, 503-509.

Holst, L. (2007). Counts of failure strings in certain Bernoulli sequences. J. Appl. Prob. 44, 824-830.

Joffe, A., Marchand, E., Perron, F. And Popadiuk, P. (2004). On sums of products of Bernoulli variables and random permutations. J. Theoret. Prob. 17, 285-292.

Knuth, D. (1992). Two notes on notation. Amer. Math. Monthly 99, 403-422.

Mori, T. F. (2001). On the distribution of sums of overlapping products. Acta Sci. Math. 67, 833-841.

Sethuraman, J. and Sethuraman, S. (2004). On counts of Bernoulli strings and connections to rank orders and random permutations. In A Festschrift for Herman Rubin (IMS Lecture Notes Monogr. Ser. 45), Institute of Mathematical Statistics, Beachwood, OH, pp. 140-152. 